\title{
Low Cost Ultrasonic Based Wide Detection Range Smart Walking Stick for Visually Impaired
}

\author{
De Alwis D and Samarawickrama YC
}

Faculty of Engineering and Technology, Colombo International Nautical Engineering College (CINEC), Malabe, Sri Lanka

\begin{abstract}
Blindness is a medical condition that requires approaching life in different ways. Unfortunately blindness still lacks proper care in the world. Many investigations have been carried out to facilitate the self-sufficient movements for the blind. The basic elementary instrument used is the traditional white cane. Many electronic travel aids have been introduced to the global market. However, more consideration is required for drawbacks of high cost, less user friendliness and less accuracy in these electronic travelling aids. Overcoming the challenges for visually impaired victims this article proposes a low cost ultrasonic based smart blind walking stick. This is a technological upgrade to the traditional white cane normally used by the blind. This device assists the victims to walk more confidently and independently. Most of the visually impaired belong to the low income community. Hence the main consideration was developing this device with Low cost and best achievable performance. This design for the visually impaired is integrated with a water sensor and ultrasonic obstacle detection sensors. The different obstacle conditions namely; mud puddles, low lying objects, ascending stairs, descending stairs and head high obstacles can be effectively and accurately detected. Early warning of danger is conveyed to the user due to the feature of wide obstacle detection range. The user is alerted by unique fingertip vibrations and sound patterns corresponding to the relevant user alert. The innovative design of the stick enables more compatibility and a wider obstacle detection range for the device with high accuracy. The low cost achievement of this design is outstanding with a total cost of less than 30 US dollars which can be further reduced in mass production.
\end{abstract}

KEYWORDS: Electronic travelling aid, low cost, ultrasonic sensing, obstacle detection, vibration alerts 


\section{INTRODUCTION}

Blindness is a medical condition that requires proper consideration and care since it requires approaching life in new ways. Most victims encode spatial relationships to form representations of the real world while adapting to newer skills and techniques. These techniques include learning Braille, following trained dogs and installing rails in bathrooms and organizing essential items for quick grasp. However, these approaches would be quite expensive.

Considering research studies and investigations carried out to enable self-sufficient movements for the blind, Electronic Travelling Aids (ETAs) are significant. These ETAs have the ability to serve the blind by guiding towards destination and detecting the obstructions ahead of the user of the device. These habitual technologies used are cameras, infra-red sensors, ultrasonic sensors and GPS. However the expensiveness of the ETAs available in the global market is a major drawback since most of the blind people are prevailing from low income backgrounds.

Therefore, blind victims currently using the basic, straight forward and elementary device; the traditional white cane although many electronic travel aids (ETA) have been introduced. Furthermore, proper consideration should be focused on enhancing the compatibility and the accuracy of current market designs in addition to the high cost of them $(\mathrm{Hu}$ et al., 2009; Saaid et al., 2009).

The aim of this design was to develop a practically useful and beneficial walking stick for visually impaired people at an outstandingly low cost. This fulfills the requirements of independent mobility and affordable price identified as a prudent requirement for the current market. The design would make the visually impaired more emotionally and intellectually independent helping to deal with life with more confidence.
Considering the objectives of this design, interests were specially focused on successful identification and alerting the user about different kinds of obstacles such as low-lying obstacle, head-high obstacles, ascending stairs, descending stairs and mud puddles on the route of the user.

\section{BACKGROUND}

About 286 million people worldwide are visually impaired and out of it, approximately 37 million people are blind and 246 million have low vision. One in three senior citizens over age 65 faces sight loss. Approximately 19 million children are visually impaired, out of which, 1.4 million suffer from blindness which cannot be altered.

Out of that around $90 \%$ of visually impaired people are over the age of 65 . Out of all the visually impaired population, $65 \%$ are aged 50 and older, while this age group contains about 20 $\%$ of the world's population. With the increasing elderly population in majority of countries, the quantity of people with the threat of visual impairment due to chronic eye diseases and ageing processes will be more (American Foundation for the Blind, 1977).

The standard ETAs that use cameras usually depend on image processing technology and transfiguration of obtained images. The user feedback is provided as recorded audio messages, vibrations and other note impressions. ETAs which apply GPS technology generally provide information to the user of their current location guiding the users to their preferable destinations. Another type of the ETAs contains arrays of sensor networks. This is an installed system of network that enabled the cane to read their proximities and deviate from obstacles. Other applications of ETAs incorporate immediate obstacle detection technology by distance using simple sensors like ultrasonic and infrared (Shoval et al., 2003; Rey et al., 2015). 


\section{MATERIAL \& METHODS}

This fully functional Smart walking stick is designed successfully with the capability to detect mostly encountered obstacle that lies ahead. It will not only detect human height obstacles but is also capable of differentiating the different types of obstacles ahead and warn the user with vibrations in the users' fingertips and appropriate audio feedback. The selection of components is significant providing efficient results at an outstandingly low cost.

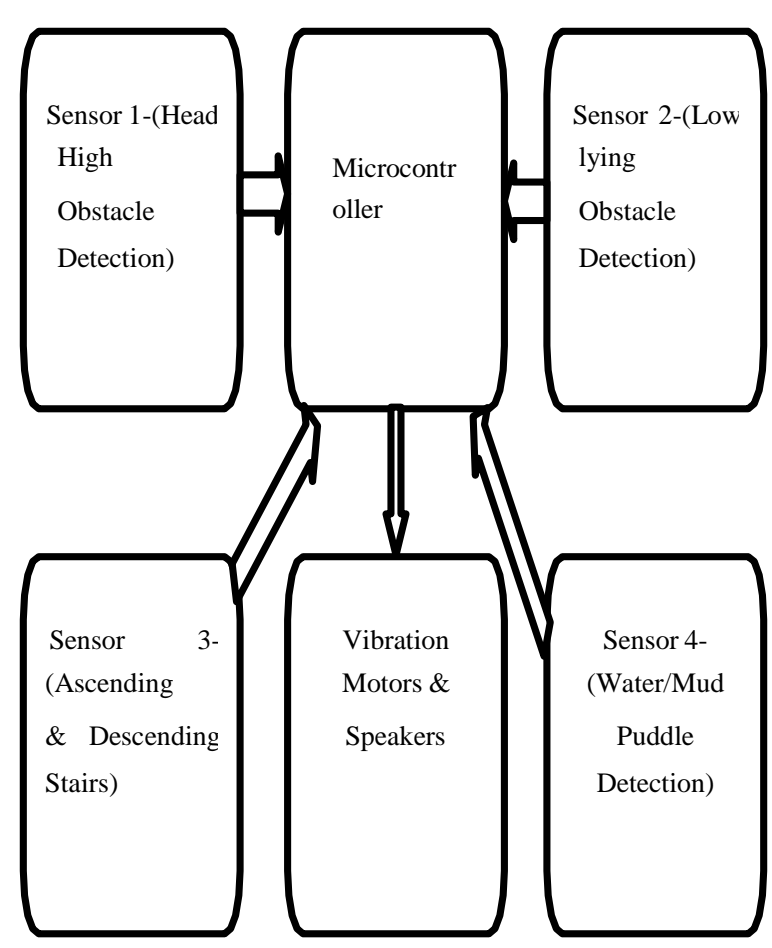

Figure 1. Basic Block Diagram of the design

Choosing the appropriate sensor depended on factors like cost, detection range and the required functionality. Ultrasonic sensors transmission and reception used ultrasound waves that could detect a range from $5 \mathrm{~cm}$ to $10 \mathrm{~m}$ with a very high accuracy. Low cost of the ultrasonic sensors available in the market contributed for the cost effectiveness of the final design. Ultrasonic sensors provided with accurate results for closer objects and were well suited for the required functionality.
The ability of ultrasonic sensors to detect clear, transparent and shiny targets as well as solid dark and opaque materials was also a positive feature of the sensors. Detection of the obstacles is not obstructed by dust or dirt and not affected by low light conditions. Hence, the device is usable at night time without issues. This feature of using the device during the night time without malfunctioning is also significant.

The different types of environment obstacles are identified and user alerts are provided as in the block diagram of the system in figure 1 . Ultrasonic sensor 1 is focused to detect head high obstacles and ultrasonic sensor 2 to detect low lying obstacles. Ultrasonic sensor 3 aimed towards the ground assists in detecting and differentiating between ascending and descending stairs. The Ultrasonic sensor 3 was effectively used to differentiate between flat terrain, ascending stairs and descending stairs as comparing the distances measured by the sensor. When the walking stick is facing an ascending staircase the distance measured by the sensor is less compared to the distance measured in a flat terrain. However, when the walking stick is

Facing a descending staircase the distance measured by the sensor is high compared to the distance measured in a flat terrain. Water sensor 4 is used for detection of mud puddles.

All sensor readings are analyzed by the microcontroller to measure the corresponding distance to the obstacle. Then alerts are provided appropriately to the user as fingertip vibrations and sound patterns. The mechanical structure of the device is highly compatible and sensors are placed affectively to detect obstacles in a wide range. Aluminum is a good light weighted metal and is used to build the device.

The physical structure is built with proper consideration during the design. This is where the length of the cane comes into major consideration. Relying on data that average human structure standing erect ranged from a height of 1.4 meters and 1.9 meters, the structure 
was made height adjustable in this range. Mobility was enhanced with a free wheel. This incorporation of wheels into the structure provided stability and mobility while it reduced friction.

\subsection{Detection of ascending and descending stair obstacles}

The ultrasonic sensor 3 was first used to determine the distance to the flat terrain as illustrated in figure 2(c). The distance the beam travels once it approaches the upstairs as in figure 2(a) is shorter than the distance the beam it travels on the flat terrain since the beam approaches an obstacle at a much shorter distance. The distance the beam travels once it approaches the downstairs as in figure 2(b) is longer than the distance the beam it travels on the flat terrain.

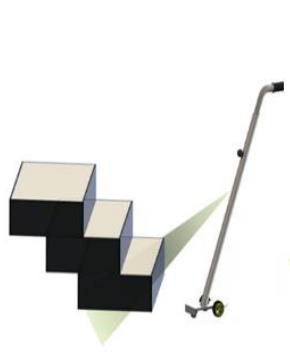

(a)

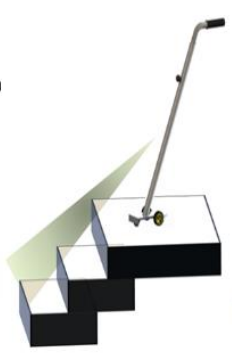

(b)

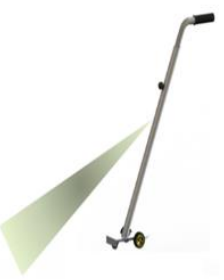

(c)
Figure 2. Detection of ascending and descending stair obstacles

(a) Ascending stairs detection

(b) Descending stairs detection

(c) Flat terrain

\subsection{Low lying obstacle Detection}

The ultrasonic sensor 2 detects low-lying obstacles as in figure 3.It is also used as a verification sensor to alarm the user that the travelling direction should be altered.

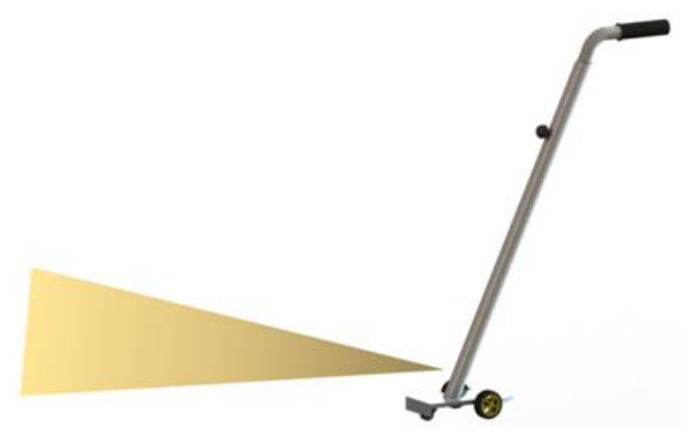

Figure 3. Low lying obstacle detection

\subsection{Overhead and head-high level obstacle Detection}

Ultrasonic sensor 1 detects branches of trees, banners, inward tilting walls and other similar obstacles. These could be classified as head high or overhead obstacles. The first ultrasonic sensor was fasted onto a servomotor that would rotate onto a pre-defined angle detecting the relevant obstacles as in figure 4 .

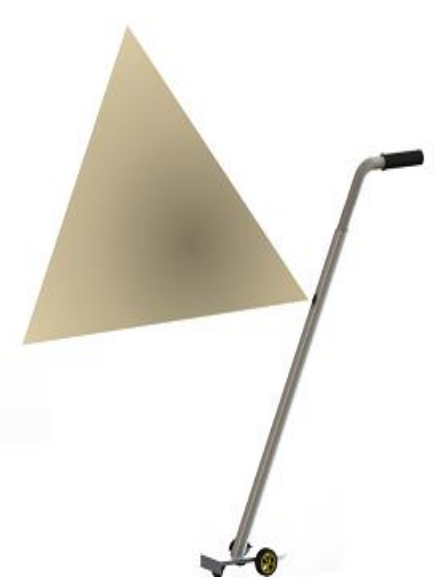

Figure 4. Detection of Overhead and head high obstacles

The servomotor was mounted perpendicular to the walking stick. The ultrasonic sensor 1 was mounted on the servo motor. The servo motor rotation covers up a height of 4 feet to 6 feet in the vertical path. 


\subsection{Water/ mud puddle detection}

The water sensor is attached to the lower section of the device as in figure 5. It alerts the user once the probes of the sensor come to contact with water or a mud puddle. The sensor measures the conductivity between the probes of the sensor. The water or mud puddle detection function was functioning properly in rain also.

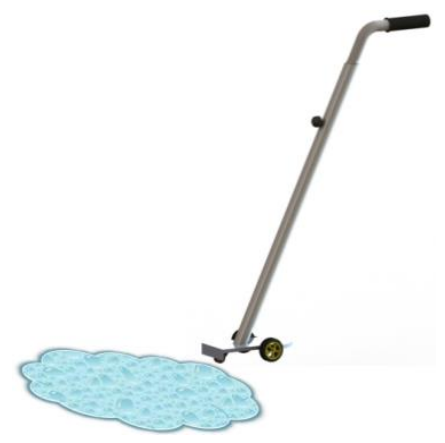

Figure 5. Water/ mud puddle detection

\subsection{Differentiation ascending and descending stairs}

The ultrasonic sensor 3 measures the distance to the flat terrain and saves this value as D in the program in the microcontroller. The measurements on sensor 3 denoted as D1 is compared with the value $\mathrm{D}$ in real-time.

If $\mathrm{D} 1$ is higher than $15 \mathrm{~cm}$ below the saved value $\mathrm{D}$,

$$
\text { D_1 } 1>\text { D-15 }
$$

Ascending stairs are identified.

If $\mathrm{D} 1$ is lower than $15 \mathrm{~cm}$ above the saved value $\mathrm{D}$,

$$
\text { D_1 }>\text { D+15 }
$$

Descending stairs are identified. After successful differentiation of ascending and descending stairs relevant vibration pattern and audio feedback is provided to user.

\section{RESULTS \& DISCUSSION}

The design was tested in the practical environment for detection of head high obstacles, low lying obstacles, ascending stairs, descending stairs and mud puddles.

The following figures illustrate the distance variations measured by the device for the different types of obstacles detected at several different landscaping. Linear lines have been approximated to denote the variation patterns.

The corresponding user alert is conveyed to the user after identifying these variation patterns of the distance. Each obstacle was tested with the targeted sensor individually and also with the all the sensors running concurrently.

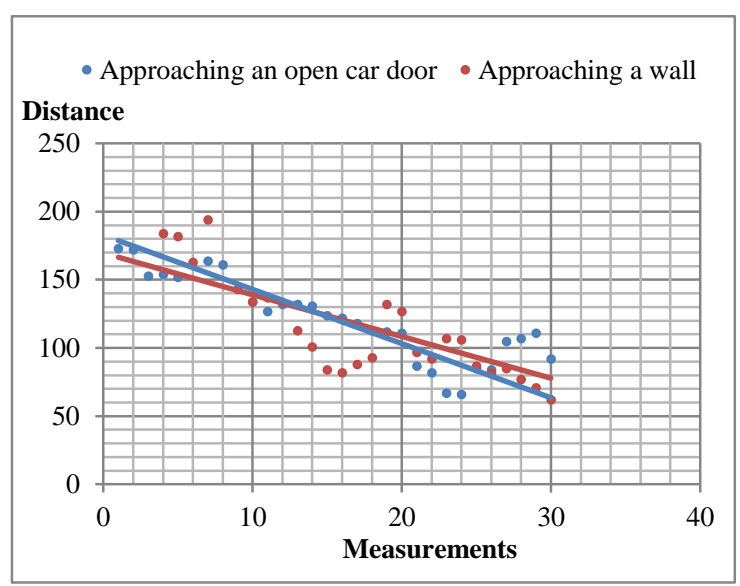

Figure 6. Variation pattern for overhead obstacles

Figure 6 illustrates the variation pattern for overhead obstacles obtained by ultrasonic sensor 1.

The downward sloping line of the graph indicates the decreasing of distance measured from the sensor to the obstacle. This implies the device is approaching an overhead/ head-high level obstacle.

The device was also tested against a blind wall successfully. This detected and signaled as a 
direction blockage. The variation patterns indicated in figure 6 confirm the presence of a head-high obstacle ahead of the user.

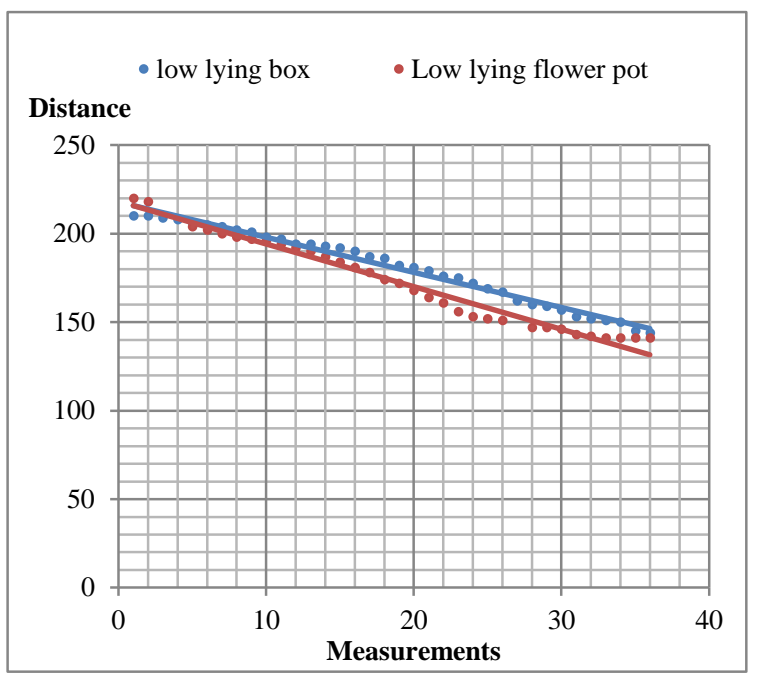

Figure 7. Variation pattern for Low lying obstacles

Figure 7 illustrate the variation patterns from ultrasonic sensor 2 against low lying obstacles. The device with a downward sloping graph indicates the device is approaching a low lying object.

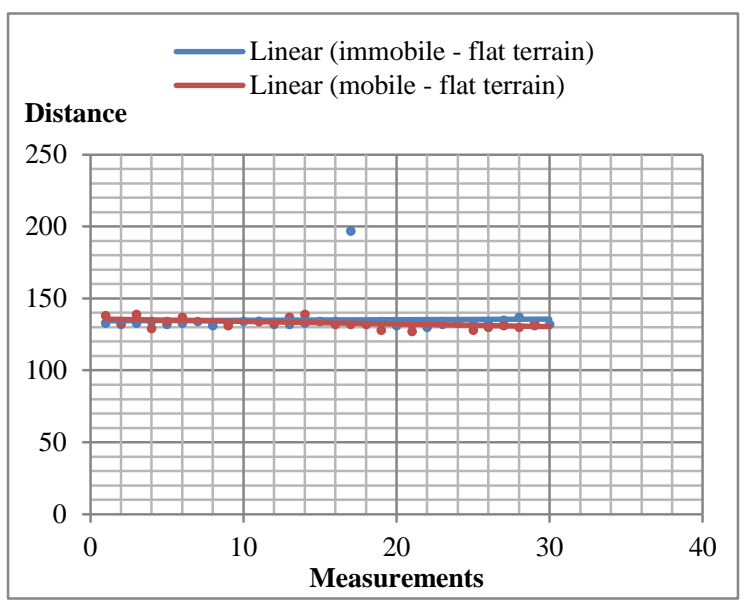

Figure 8. Variation pattern in the flat terrain

Figure .8 illustrates the measurements recorded by ultrasonic sensor 3 in mobile and immobile conditions on flat terrain.

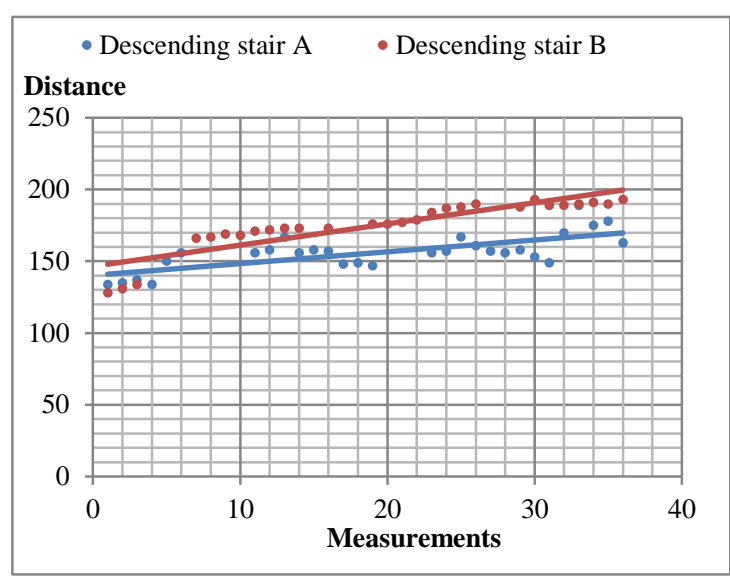

Figure 9. Variation pattern for descending stairs

The device was then tested against descending staircases of two heights. Figure .9 illustrates the measurements on ultrasonic sensor 3 at a descending staircase. The measurements and variation pattern on figure .9 with an upward sloping graph proved the device approached a descending staircase.

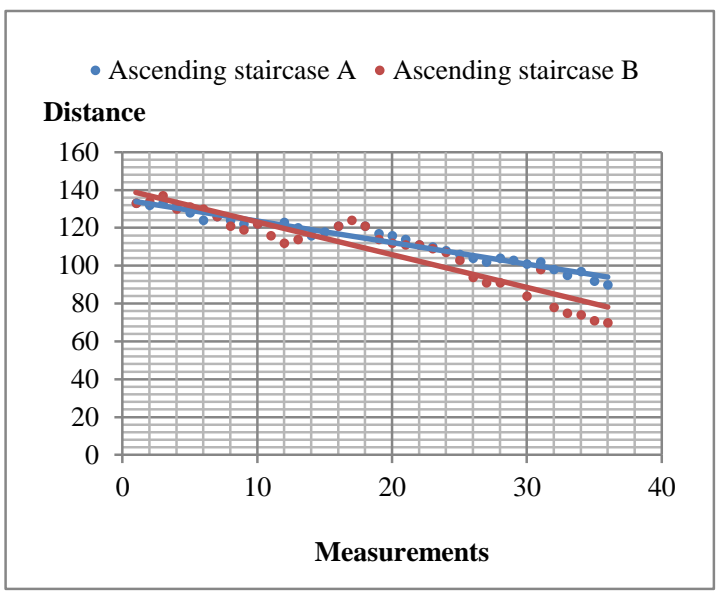

Figure 10. Variation pattern for ascending stairs

Ultrasonic Sensor 3 was then tested against ascending staircases of two heights. Figure. 10 illustrates the measurements recorded for ascending stairs. Results illustrated in figure 10 proved a downward sloping graph indicting the device was approaching an ascending staircase. 
Even though the Ultrasonic sensor 2 was for detecting low-lying objects, it also read the lowest step of an ascending staircase. Boxes were kept on the floor for low lying objects. The confusion between ascending staircases and lowlying objects were avoided by programming the vibrators with unique vibration patterns. The water sensor detected mud puddles and water effectively by providing a low analog output voltage compared to the normal conditions.

Most importantly, the low cost achievement is outstanding since the total cost of the design is less than 4000 LKRs as presented in the table 1.This design fulfills a timely requirement fulfilling real life problems of visually impaired community. Hearing and spatial sensing has been the leading and finest senses available to visually impaired users. These two senses have not been able to substitute vision, but have been able to capture huge environmental data. The most touch sensitive areas of the human body are finger tips, upper lip, cheeks, palm, forehead and foot respectively. The main advantage of using tactile vibrations on the fingertips of the user is that it allows the user to grasp environmental cues while travelling independently.

Table 1. Total cost of the Design

\begin{tabular}{|l|l|l|}
\hline Item Purchased & No & $\begin{array}{l}\text { Total } \\
\text { (LKR) }\end{array}$ \\
\hline Moisture Water Sensor & 1 & 142.56 \\
\hline Ultrasonic Module & 3 & 427.68 \\
\hline AT mega Arduino & 1 & 1094.4 \\
\hline Vibrating motor & 4 & 483.84 \\
\hline 9G micro servo motor & 1 & 226.08 \\
\hline Jumper Cable & 1 & 437.76 \\
\hline Solder less breadboard & 1 & 109.44 \\
\hline Bicycle grip & 1 & 50.00 \\
\hline Prototype structure & 1 & 700.00 \\
\hline Total Cost & $\mathbf{3 6 7 1 . 7 6}$ LKRs \\
\hline
\end{tabular}

\section{CONCLUSION/FURTHER WORK}

Analyzing the results, we can conclude that the objectives of the study were successfully achieved. Successful identification and alerting the user of different types of obstacles with high accuracy is a significant achievement for an outstandingly low cost.

Composing the structure into a foldable structure without any external tool would further ease storage when the device is not in use. The device could also be reinforced with a nylon strip on the grip that would be wrapped around the user's wrist allowing the device to travel steadily with user.

The device could also be integrated with audio feedback system. Toggling between audio and tactile vibrations would bring out a change in the users everyday usage pattern.

Ability of toggling between outdoor and indoor environment conditions would be highly appreciated. That is the sensors could be programmed to detect at a larger range when the user is outdoors and at a smaller range when the user is indoors by simply swapping between two buttons.

To have a better technological upgrade of the device, the device could also be integrated with a low cost camera like the monocular camera to identify and detect yellow lines (Zebra crossings) on the road. Alerting the user when the user is on a yellow line would further add independence and confidence. Users will also be a lot more comfortable when using the device.

If the system was to be upgraded in an advanced manner, GPS navigation system could be integrated with the system. As the user is visually impaired and cannot read or enter destinations on GPS, this system could be programmed to operate on voice command of the user. Hence this system will be working on audio cues at the ears of the users which directs user to the preferred destination. 


\section{REFERENCES}

American Foundation for the Blind, Journal of Visual Impairment \& Blindness, USA. American Foundation for the Blind, Information Department, Publications Division. 1977.

HU L, LOU W, SONG R, GAO C \& LI X. A novel design of micro - magnetic sensor guidance system for the blind. $4^{\text {th }}$ IEEE international conference on nano / micro engineered and molecular systems. 2009.

SAAID MF, ISMAIL I \& NOOR MZH. Radio Frequency Identification Walking Stick (RFIWS): A Device for the Blind. $5^{\text {th }}$ International Colloquium on Signal Processing \& its Applications. 2009.

SHOVAL S, ULRICH I \& BORENSTEIN J. Robotics-Based Obstacle-Avoidance Systems for the Blind and Visually Impaired: NavBelt and the GuideCane. In: IEEE Robotics \& Automation Magazine. 2003; 12-19.

REY M, HERTZOG I, KAGAMI N \& NEDEL L. Blind Guardian: A Sonar-Based Solution for Avoiding Collisions with the Real World. 2015 XVII Symposium on Virtual and Augmented Reality. 2015. 